\title{
Consideracões sobre Abelisauridae (Dinosauria: Theropoda) e o registro brasileiro
}

\author{
Considerations about Abelisauridae (Dinosauria: Theropoda) and the Brazillan registry
}

\author{
Rafael Delcourt ${ }^{1}$, Natan Santos Brilhante ${ }^{1}$, Fresia Ricardi-Branco ${ }^{2}$ \\ 1- Programa de Pós-Graduação em Geociências, Instituto de Geociências, Universidade Estadual de Campinas, Campinas, SP. \\ 2- Programas de Pós-Graduação em Geociências e Ensino e História de Clências da Terra, Instituto de Geociências, Universidade Estadual de Campinas, Campinas, SP. \\ E-MAL::RAFAEL.DELCOURT@GMAL.COM, NATAN.BIOLOGIA@GMALl.COM, FRESIA@IGE.UNICAMP.BR
}

\begin{abstract}
Abelisauridae is a broad dinosaur family of Theropoda that includes large extinct carnivores as well as extant birds. The distribution is mostly in the southern hemisphere, although some specimens are found in Europe. The phylogenetic relationships of the abelisaurids have been improved, but there is still some disagreement regarding the inclusion of some groups in the family, which deserve attention. The body plan of the abelisaurids is conservative among the group, but they have a large cranial disparity. They also tend to develop cranial structures such as horns, thick skull bones, and cornified covering. The variety of cranial soft tissues associated with skeletal features suggests that abelisaurids may have had low-stress headbutting behavior. The Brazilian record is still scarce, and there are only two known species from different geological units. Even so, it is possible to observe that the abelisaurid diversity in Brazil was large.
\end{abstract}

Resumo: Abelisauridae é uma ampla família de dinossauros do grupo Theropoda, que inclui grandes animais carnívoros extintos bem como as aves dos dias atuais. Sua distribuição é majoritariamente austral, mas alguns espécimes são oriundos da Europa. As relações filogenéticas dos abelissaurídeos têm se tornado refinadas, mas ainda existem discordâncias quanto ao posicionamento de grupos incluídos na família que merecem atenção. 0 plano corporal dos abelissaurídeos é conservador entre 0 grupo, mas apresenta grande disparidade morfológica no crânio. Eles também possuem tendência a desenvolver estruturas cranianas que variam entre chifres, espessamentos do crânio e coberturas córneas. A variedade de tecido mole craniano associado à estrutura esquelética sugere que os abelissaurídeos poderiam apresentar comportamento de marradas de baixo impacto. 0 registro brasileiro de abelissaurídeos ainda é escasso e são reconhecidas apenas duas espécies em diferentes unidades geológicas. Ainda assim, é possível verificar que a diversidade desse grupo no Brasil foi grande.
Citation/Citação: Delcourt, R., BriIhante, N. S., \& Ricardi-Branco, F. (2020). Considerações sobre Abelisauridae (Dinosauria: Theropoda) e o registro brasileiro. Terræ Didatica, 16, 1-13, e020017. doi:10.20396/ td.v16i0.8656403

Keywords: Abelisaurids. Theropods. Evolution. Fossils. Brazil.

Palavras-chave: Abelissaurídeos. Terópodes. Evolução. Fósseis. Brasil.

\section{Manuscript/Manuscrito:}

Received/Recebido: 28/08/2019

Revised/Corrigido: 31/03/2020

Accepted/Aceito: 23/04/2020

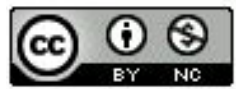

\section{Introdução}

Abelisauridae constitui uma ampla família de dinossauros do grupo Theropoda, que por sua vez inclui animais originados a partir do Período Triássico (primeiros dinossauros) até os dias atuais (aves mais recentes) (Delcourt, 2018, Langer et al., 2017, Sereno, 1999). Os abelissaurídeos tiveram sua origem a partir do período Jurássico Médio no Hemisfério Sul, diversificando durante o Cretáceo Superior até o final período Cretáceo, como mostram os fósseis recuperados na América do Sul, África, Madagascar, Índia/Paquistão e Europa (Carrano \& Sampson, 2008, Delcourt, 2018, Novas et al., 2013, Pol \& Rauhut, 2012).

$\mathrm{Na}$ América do Sul, o registro desse grupo é bem conhecido principalmente na Argentina, onde ocorrem as espécies mais antigas (i.e., Eoabelisaurus mefi Pol \& Rauhut, 2012, do Aaleniano-Bajociano, Jurássico Médio) até as mais recentes (e.g. Carnotaurus sastrei Bonaparte 1985, do Maastrichtiano, Cretáceo Superior). No que diz respeito ao Brasil, o conhecimento desse grupo ainda é escasso, sendo reconhecidas, até o momento, apenas duas espécies: Pycnonemosaurus nevesi Kellner \& Campos, 2002 (ver também Delcourt, 2017) e Thanos simonattoi Delcourt \& Iori, 2018. Os demais registros brasileiros limitam-se a fósseis fragmentários sem informação suficiente para estabelecer novas espécies (e.g. Delcourt \& Grillo, 2018, Mendez et al., 2014, Novas et al., 2008, Sales et al., 2018).

Apesar de o registro dos abelissaurídeos ser composto por espécimes com alto grau de completude, apresentando até mesmo impressão de pele (Bonaparte et al., 1990) e sendo considerados 
os principais predadores do hemisfério sul no final do Cretáceo (Delcourt, 2018), esse grupo de terópodes não tem recebido a mesma atenção que seus relativos cinematográficos como Tyrannosaurus rex e Velociraptor mongoliensis. Na presente contribuição será revisto o estado da arte do registro da família Abelisauridae em terras brasileiras (ver Tab. 1), apresentando-se um etograma visual do grupo a partir de informações anteriormente publicadas.

\section{Relações filogenéticas}

As relações de parentesco entre os abelissaurídeos têm sido refinadas com diversos trabalhos descritivos e filogenéticos. Inicialmente, o entendimento do grupo era limitado, quando foi descrito o holótipo da família, Abelisaurus comahuensis Bonaparte \& Novas, 1985, pois eram realizadas comparações morfológicas com outros terópodes filogeneticamente distantes. No entanto, a partir de novas descobertas de diferentes abelissaurídeos, sobretudo na Argentina, as descrições e o entendimento do grupo passaram a ser mais refinados (Bonaparte, 1991, Carrano \& Sampson, 2008) e, atualmente, as relações dos táxons internos têm sido mais estáveis nos últimos trabalhos. Em outras palavras, a compreensão da relação de parentesco entre os abelissaurídeos tornou-se mais sólida em pesquisas que utilizaram diferentes bases de dados evidenciando uma maior coerência nas topologias (Fig. 1) (e.g. Canale et al., 2009, Carrano \& Sampson, 2008, Delcourt, 2017, Filippi et al., 2016, Longrich et al., 2017, Pol \& Rauhut, 2012; Rauhut \& Carrano, 2016, Tortosa et al., 2014).

Recentemente, Wang et al. (2017) publicaram uma revisão filogenética de Ceratosauria (clado que inclui Abelisauridae e outros grupos de terópodes), apresentando resultados heterodoxos em relação aos trabalhos de sistemática já publicados. Basicamente, a relação filogenética tradicional entre Ceratosauria dá-se pela conformidade: (Ceratosauria basais (Noasauridae, Abelisauridae)) (e.g. Carrano \& Sampson, 2008). No entanto, Wang et al. (2017) encontraram a seguinte topologia: (Noasauridae (Ceratosauridae, Abelisauridae)). Resultado esse respaldado por uma ampla base de dados contendo um $\mathrm{N}$ amostral de 214 dinossauros analisados e 744 caracteres morfológicos listados, sendo considerada uma das maiores bases de dados para análise de Ceratosauria até o presente.

No entanto, conforme mostrado por Grillo \& Delcourt (2017) a construção de certos caracteres morfológicos pode estar baseada em premissas equivocadas, como por exemplo em descrição de proporções de partes ósseas ontogeneticamente isométricas. Dentro desse contexto, esses autores revisaram os caracteres de matrizes já publicadas (e.g. Carrano \& Sampson, 2008, Filippi et al., 2016), sugerindo que alguns caracteres deveriam ser mudados ou mesmo excluídos (e.g. caracteres sem verificação do desenvolvimento alométrico do plano corporal). Dessa forma, a matriz de dados publicada por Wang et al. (2017) deve ser considerada cuidadosamente, e sua hipótese filogenética tomada com precaução, considerando os demais trabalhos que concordam entre si na topologia geral (Canale et al., 2009, Carrano \& Sampson, 2008, Delcourt \& Iori, 2018, Pol \& Rauhut, 2012).

\section{Plano corporal de Abelisauridae}

O plano corporal dos abelissaurídeos difere consideravelmente dos demais dinossauros terópodes, mas ainda assim continua a obedecer ao padrão sugerido por Lockley et al. (2008): cabeças bem desenvolvidas, com braços e pescoços curtos (Fig. 2). O padrão é observado em diversos terópodes tais como Tyrannosaurus rex (Brochu, 2003), Giganotosaurus carolini Coria \& Salgado, 1995 e Allosaurus fragilis (Madsen, 1976). Segundo a tese de Delcourt (2016), os abelissaurídeos possuíam, ao menos no crânio, a maior disparidade morfológica dentre todos os terópodes não avianos, o que contribui para seu sucesso evolutivo no transcurso da Era Mesozoica (Delcourt, 2016, 2018).

No caso dos abelissaurídeos, o crânio foi bem característico, apresentando braquirostria ao longo da filogenia. Ou seja, o crânio se tornou mais curto nas espécies mais derivadas (e.g. Carnotaurus satrei) que nas espécies mais basais (e.g. Rugops primus) (Delcourt, 2018). Além disso, os abelissaurídeos mostraram tendência a desenvolver estruturas cranianas como chifres (i.e. Majungasaurus crenatissimus e Carnotaurus) (Bonaparte, 1985, Sampson \& Witmer, 2007), região dorsal do crânio inflada (i.e. ossos pós-orbitais; Skorpiovenator bustingorryi) (Canale et al., 2009), e mesmo tecidos córneos nos crânios (Delcourt, 2018). As características, embora estejam concentradas na região dorsal da cabeça, não possuem homologia (Sampson \& Witmer, 2007). Assim, as estruturas cranianas não estavam presentes no seu ancestral comum.

No que diz respeito ao tecido córneo nos crânios dos abelissaurídeos, Delcourt (2018) propõe 

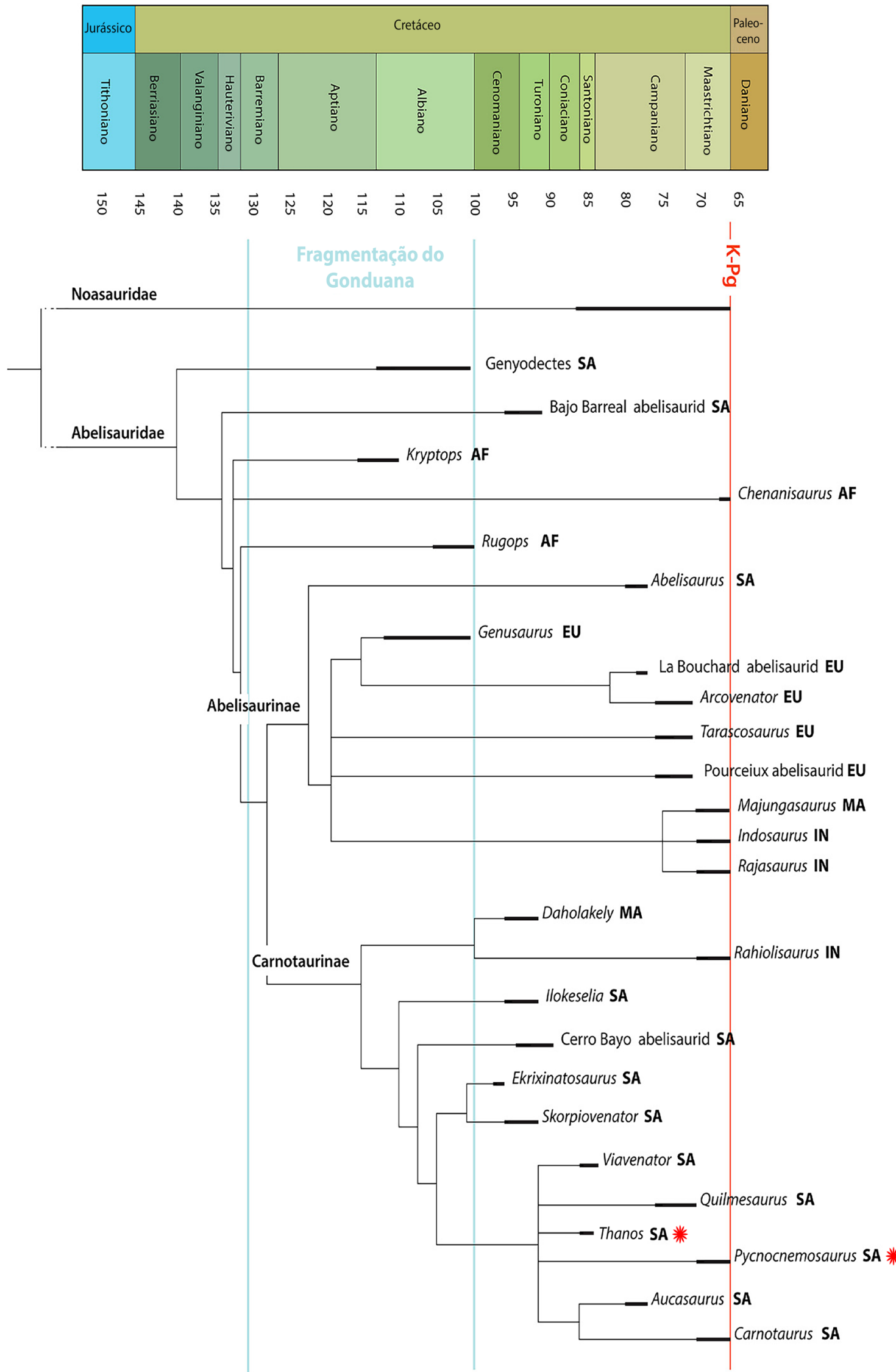

Figura 1. Relações filogenéticas entre Abelisauridae. O diagrama mostra as principais espécies de acordo com o nível de parentesco, idade em milhões de anos e localidades de ocorrência. As espécies marcadas com estrelas vermeIhas foram encontradas em unidades geológicas brasileiras. Abreviações: AF, África; EU, Europa; IN, Índia; MA, Madagascar; AS, América do Sul. Modificado de Longrich et al. (2017) 
que existiu uma hierarquização na textura do tecido mole que reveste o crânio até culminar no desenvolvimento de uma superfície córnea observada em alguns táxons. Ainda seguindo os trabalhos de Hieronymus et al. (2009) e Carr et al. (2017), Delcourt (2018) indicou que a superfície perfurada dos ossos nasais de Rugops foi recoberta por escamas sobrepostas, tais como aquelas observadas em crocodilos atuais e répteis atuais. Esse mesmo tipo de cobertura estaria presente nos ossos laterais dos crânios de todos os abelissaurídeos (e.g. ossos maxilares e jugais). Já a textura papilar observada na região posterior dos processos de articulação entre os pré-maxilares e os nasais de Rugops, e nos nasais de Abelisaurus, sugerem que essas regiões eram cobertas por escamas mais reforçadas chamadas de armour-like dermis (pele de armadura) (Delcourt, 2018). Por sua vez, a superfície dorsal dos crânios dos táxons mais derivados (e.g. ossos nasais, frontais, regiões dorsais dos lacrimais e pós-orbitais de Carnotaurus) era revestida por tecidos córneos, como observado no crânio do boi-almiscarado atual (Ovibos moschatus) (Fig. 3). Essas estruturas associadas com as características dos membros posteriores e vértebras permitem inferir os comportamentos descritos a seguir.

Os membros anteriores dos abelissaurídeos foram extremamente reduzidos a partir das regiões distais, perdendo a possibilidade de agarrar (Agnolin \& Chiarelli, 2010). Espécies basais, como Eoabelisaurus, apresentam as ungueais das mãos reduzidas (Pol \& Rauhut, 2012), enquanto que em outros táxons mais derivados, como Carnotaurus, Majungasaurus e Aucasaurus garridoi, essas estruturas estão ausentes nos dedos I e IV das mãos (Agnolin \& Chiarelli, 2010, Burch \& Carrano, 2012, Coria et al., 2002). Apesar dessa redução dos membros anteriores, a cabeça do úmero em abelissaurídeos era arredondada, sugerindo grande mobilidade na região proximal do braço (Burch, 2017, Gianechini et al., 2015). A redução dos membros anteriores em abelissaurídeos não indica uma relação homóloga com a redução em Tyrannosauridae e Alvarezsauridae.

Os membros posteriores dos abelissaurídeos são mais conhecidos pelo desenvolvimento da crista cnemial da tíbia. Nesse processo anterodorsal da tíbia, estavam inseridos os principais músculos extensores do joelho (i.e., iliotibilaes) (Romer, 1923). O tamanho avantajado e a inclinação dorsal da crista cnemial sugerem que os músculos extensores do tornozelo e flexores dos dígitos

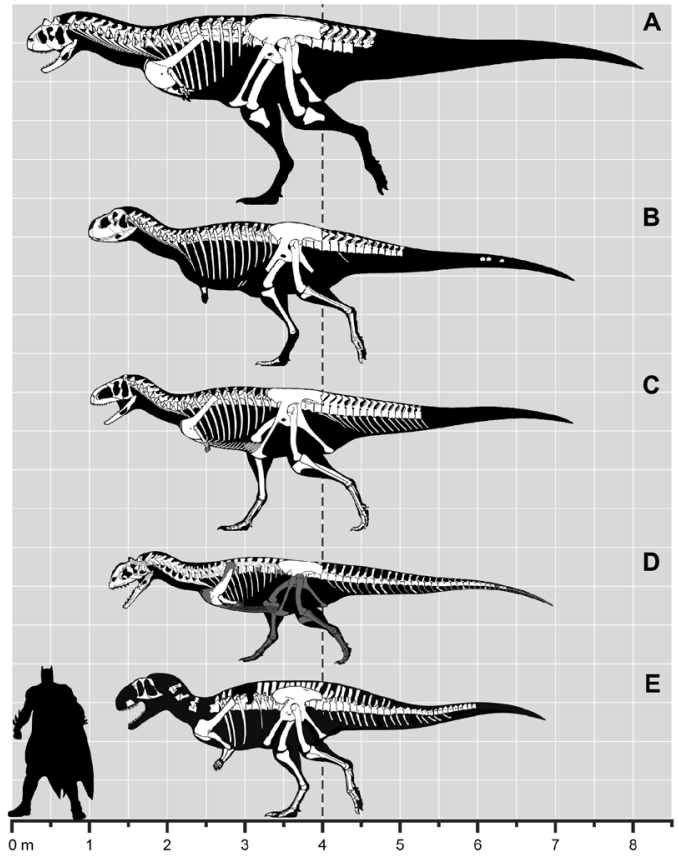

Figura 2. Reconstruções de esqueletos de Abelisauridae mostrando o tamanho corporal em metros. A, Carnotaurus sastrei; B, Skorpiovenator bustingorryi; C, Aucasaurus garridoi; D, Majungasaurus crenatissimus; E, Eoabelisaurus mefi. Reconstruções dos esqueletos modificadas de A: Bonaparte et al. (1990); B e C: Paul (2010); D: Scott Hartman; E: Pol \& Rauhut (2012). Figura geral modificada de Grillo \& Delcourt (2017)

A

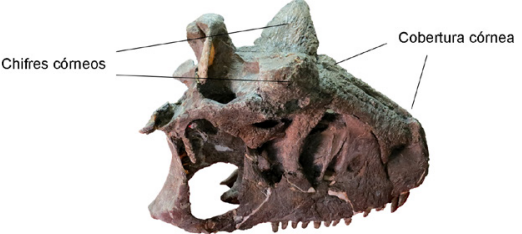

B Cobertura de escamas cobertura de pele C

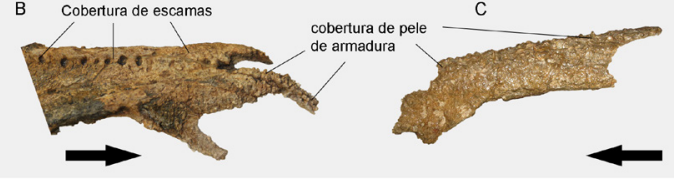

E
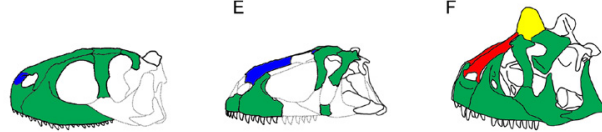

Escamas

Figura 3. Detalhes das estruturas cranianas de Abelissauridae mostrando as inferências de tecido mole. A, crânio de Carnotaurus sastrei; B, vista lateral direita dos nasais de Rugops primus; C, vista lateral esquerda dos nasais de Abelisaurus comahuensis; E - F, vistas laterais esquerdas dos crânios de Rugops, Abelisaurus e Carnotaurus mostrando as coberturas de tecido mole nos crânios. As setas pretas em B e C mostram o sentido anterior do crânio. Figura sem escala e modificada de Delcourt (2018) 
eram largos, aumentando sua capacidade de força para movimentos cursoriais (Delcourt, 2018). Mesmo que tivessem habilidade cursorial acima dos demais terópodes de grande porte (Persons \& Currie, 2011), os abelissaurídeos deveriam possuir uma capacidade de aceleração inicial maior que a velocidade de corrida, considerando o tamanho pequeno do pé comparado aos dos demais terópodes (Carrano, 2007).

As vértebras dos abelissaurídeos também diferem consideravelmente dos demais dinossauros terópodes, no que concerne aos processos presentes no arco neural. As vértebras cervicais possuíam as epipófises e espinhos neurais bem desenvolvidos, para inserção de uma forte musculatura do pescoço (Méndez, 2014). Enquanto que nas vértebras caudais a inclinação dorsal e expansão dos processos transversos sugerem que o principal músculo extensor do fêmur (i.e., caudofemoralis longus) deve ter sido mais desenvolvido do que em outros terópodes, contribuindo assim para habilidade cursorial dos abelissaurídeos (Persons \& Currie, 2011).

O tamanho corporal dos abelissaurídeos aparentemente aumentou ao longo da filogenia, de modo especial após a diminuição/extinção de Carcharodontosauridae e Spinosauridae no Turoniano (Cretáceo Superior) (Grillo \& Delcourt, 2017), ocupando o nicho ecológico de grandes predadores ao final do Cretáceo (Delcourt, 2018, Grillo \& Delcourt, 2017, Novas et al., 2013). No entanto, um fragmento de tíbia do intervalo Berriasiano-Valanginiano (Cretáceo Inferior) sugere que abelissaurídeos possam ter alcançado tamanhos relativamente grandes, com aproximadamente sete metros de comprimento, cerca de 47 milhões de anos antes da extinção desses grupos citados (Canale et al., 2017).

\section{Comportamento de Abelisauridae}

O comportamento em táxons fósseis nem sempre é fácil de deduzir. Não é possível ir a campo para observar a interação entre os animais com o ambiente e entre si. No entanto, diversas pistas podem sugerir possibilidades de comportamentos que os animais poderiam adotar. Dessa forma, a partir das análises dessas pistas e comparações com táxons atuais, é possível sugerir com alto grau de confiabilidade as possibilidades de comportamento (ver Hieronymus et al., 2009, Sakamoto, 2010, Snively, 2006).

O comportamento gregário em dinossauros foi descrito em diversos táxons (e.g. Carrano et al., 2011, Wang et al., 2017). No caso dos abelissaurídeos, vários espécimes de Majungasaurus foram encontrados associados com fósseis (costelas, arcos hemais, espinhos neurais, processos transversos e arcos neurais) que exibiam marcas de dentes de Majungasaurus, sugerindo que esse gênero possuía comportamento de canibalismo (Rogers et al., 2007). O canibalismo em Majungasaurus pode ser explicado pela escassez de recursos na Formação Maevarano, Madagascar, durante o final do Período Cretáceo (Rogers et al., 2007).

A partir das informações de tecido mole (e.g. cobertura córnea e chifres) apresentadas acima adjuntas às demais informações osteológicas, é possível inferir que os abelissaurídeos poderiam adotar comportamentos inéditos entre animais carnívoros, conforme mostrado por Delcourt (2018).

A presença de tecido córneo sobre a região dorsal do crânio, que foi inferida para espécies como Carnotaurus e Majungasaurus, está relacionada ao comportamento de marradas observado em diversos táxons atuais (e.g. Ovibos moschatus, Syncerus caffer e Buceros vigil) e extintos (Pachyrhinosaurus, Achelousaurus e Stegoceras) (Hieronymus et al., 2009, Snively $\&$ Theodor, 2011). No entanto, marradas com maior violência deveriam ser improváveis para os abelissaurídeos, uma vez que estes não possuíam ossos esponjosos espessos (como visto em Stegoceras) (Snively \& Theodor, 2011). Dessa forma, é esperado que os abelissaurídeos que possuíam cobertura córnea sobre a cabeça pudessem dar marradas e empurrões de baixa força, tal como observado em iguanas marinhas (Amblyrhynchus cristatus; Carpenter, 1967) ou atacassem com a cabeça no pescoço e flanco dos adversários (como as atuais girafas, Giraffa camelopardalis) (Snively et al., 2011) (Fig. 4). As estruturas cranianas (i.e. cobertura córnea e chifres), associadas com a presença de uma crista nucal bem desenvolvida (Sampson \& Witmer, 2007), epipófises e espinhos neurais largos que aumentam a musculatura do pescoço (Méndez, 2014, O'Connor, 2007), corroboram para a hipótese de que o complexo cérvico-cefálico de abelissaurídeos pudesse suportar o estresse de marradas. De fato, Mazzetta et al. (2009) propuseram que Carnotaurus pudesse suportar estresses de baixa energia no crânio.

O comportamento de marradas poderia ser exercido para situações variadas, tal qual sugerido para mordidas relatadas em crânios de terópodes (Tanke \& Currie, 1998), incluindo intenções de territorialismo, cortejo, brincadeira, predação, cani-

\begin{tabular}{c|c}
\hline (C) Terrae Didat. & Campinas, SP \\
\hline
\end{tabular} 


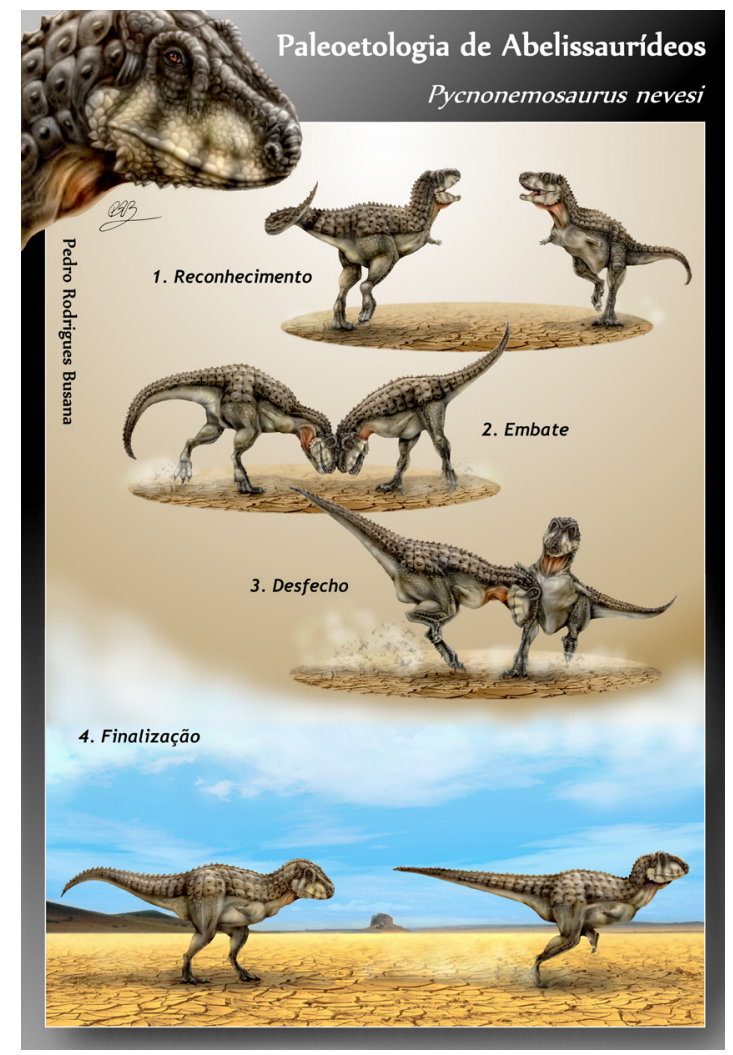

Figura 4. Etograma ilustrado de Abelisauridae mostrando as possibilidades de comportamento a partir das evidências osteológicas apresentadas no texto. Os exemplos hipotéticos são dados a partir da reconstrução em vida de Pycnonemosaurus nevesi. Arte de Pedro Rodrigues Busana

balismo e dominância de adultos. Uma vez que as estruturas cranianas que reforçaram a cabeça estavam presentes nos táxons mais basais, como Rugops, é possível que o comportamento de marrada tenha surgido no início da evolução dos abelissaurídeos. Essa hipótese pode ser testada caso um abelissaurídeo basal, como Rugops, seja encontrado e possua um complexo cérvico-cefálico bem desenvolvido como nos demais táxons mais derivados. No caso de Rugops, apenas elementos cranianos são conhecidos (Sereno et al., 2004).

Cabe ressaltar aqui que esses comportamentos e suas razões são hipóteses a partir das evidências osteológicas preservadas. As marcas e cicatrizes em fósseis devem ser sempre tomadas com cautela no momento de apresentar qualquer interpretação.

\section{0 registro brasileiro}

O registro de abelissaurídeos ao redor do globo é amplo, com vasta distribuição principalmente no Hemisfério Sul: América do Sul, África, Madagas- car, Índia/Paquistão; mas também ocorre na Europa (Carrano \& Sampson, 2008, Delcourt, 2018, Novas et al., 2013, Pol \& Rauhut, 2012). Dentre os registros conhecidos, a Argentina apresenta a maior diversidade da família (Novas et al. 2013), ao passo que o registro do grupo ainda é escassoem seu vizinho de fronteira, o Brasil (ver Tab. 1), principalmente considerando sua vastidão territorial (ver Bittencourt \& Langer, 2011).

Mesmo que escasso, comparado com outros países, o Brasil apresenta duas espécies de abelissaurídeos, além de amostras fósseis de fragmentos de ossos atribuídos ao grupo, sugerindo que esse possuía uma diversidade considerável em terras brasileiras.

A fauna dos abelissaurídeos foi mais ampla no Gondwana em relação a Laurásia, onde vários registros do grupo são dúbios (e.g. Le Loeuff \& Buffetaut, 1991, Sampson et al., 1998). Bittencourt \& Langer (2011) sugeriram que a escassez de exemplares fósseis de algumas localidades, como, por exemplo, em afloramentos que datam entre o Aptiano e o Cenomaniano do Brasil ( 125 - 93 $\mathrm{Ma}$ ), pode refletir dificuldades de coleta em regiões com densa cobertura florestal, falta de prospecção ou mesmo endemismo dos táxons.

Para o Brasil, Pycnonemosaurus nevesi Kellner \& Campos, 2002, coletado em camadas do Grupo Parecis, foi formalmente a primeira espécies de abelissaurídeo descrita, com base nos seguintes elementos ósseos parcialmente preservados: cinco coroas dentárias incompletas, duas vértebras caudais, quatro centros vertebrais caudais, um processo transverso, fragmentos de costelas, parte distal do púbis direito, tíbia direita, parte distal da fíbula direita e outros elementos que, dada a sua baixa completude, não foram passíveis de identificação. Posteriormente, Delcourt (2017) redescreveu a espécie a partir de novas comparações morfológicas e análises filogenéticas, atribuindo mais dois processos transversos como parte do holótipo de Pycnonemosaurus e excluiu as coroas dentárias. Segundo o autor, as coroas dentárias não podem pertencer ao holótipo por não haver nenhum material craniano associado e por terem sido encontradas isoladas, próximas ao local de coleta das amostras do pós-craniano. Dessa forma, Delcourt (2017) atribui as coroas dentárias, que foram descritas por Bittencourt \& Kellner (2002), à abelissaurídeos indeterminados. Até o momento, Pycnonemosaurus ainda é o abelissaurídeo mais completo do Brasil, sendo possível sugerir que o holótipo se tratava de um espécime subadulto, a partir da identificação 
das suturas no centro vertebral, bem como o não fusionamento da tíbia com o astrágalo (Delcourt, 2017). De acordo com Grillo \& Delcourt (2017), Pycnonemosaurus nevesi é o maior abelissaurídeo conhecido ( $\sim 9$ metros de comprimento), ultrapassando o comprimento de Carnotaurus $(\sim 7,8 \mathrm{~m})$ e Ekrixinatosaurus novasi $(\sim 7,5 \mathrm{~m})$.

Em 2018, Delcourt e Iori descreveram a segunda espécie de abelissaurídeo do Brasil a partir de um áxis quase completo fusionado com um intercentro, que teve as suas porções anterior e posterior independentemente coletadas e posteriormente revisadas (ver Méndez et al., 2014, Delcourt \& Iori, 2018). Thanos simonattoi representa a primeira espécie de dinossauro terópode descrita para o Grupo Bauru, e mesmo possuindo tamanho considerável dentre os abelissaurídeos $(\sim 5,5-6,5 \mathrm{~m})$, dividia o ambiente com terópodes maiores (Méndez et al. 2012, Delcourt \& Iori, 2018). A estrutura óssea de Thanos sugere que os abelissaurídeos poderiam possuir pneumaticidade mais desenvolvida do que antes pensado. No entanto, mais fósseis de abelissaurídeos precisam ser encontrados para avaliar tal hipótese.

Desde a década de 1990, o Brasil tem sido palco de diversas descobertas de fósseis atribuídos aos abelissaurídeos, recuperados de formações das bacias Bauru, Paraná e São Luís-Grajaú. Ainda que fragmentários e, muitas vezes representados por amostras isoladas, os registros sugerem que esse grupo era muito diverso no Brasil, e que correspondiam a principal fauna de dinossauros terópodes no final do período Cretáceo. O compilado dos registros a respeito dos espécimes associados aos abelissaurídeos do Brasil e do contexto deposicional os quais estavam inseridos encontram-se na Tabela 1.

Grande parte do registro de abelissaurídeos no Brasil provém das coroas dentárias coletadas em diferentes unidades litoestratigráficas (Tab. 1; Fig. 5). De maneira interessante, os dentes de abelissaurídeos possuem certa similaridade com os dentes de carcarodontossaurídeos (Canale et al., 2009, Gianechini et al., 2015) levando a interpretações errôneas quando encontrados isoladamente.

Azevedo et al. (2013) descreveram um fragmento de maxilar contendo um dente in situ (UFRJ-DG409-R), e atribuíram esse material a um carcarodontossaurídeo indeterminado da Formação Presidente Prudente (Grupo Bauru), com idade de 83-66 Ma (Campaniano- Maastrichtiano). No entanto, os carcarodontossaurídeos se tornaram raros e extintos a partir do Turoniano $(\sim 90 \mathrm{Ma})$ (Coria \& Salgado 2005, Novas et al., 2005, 2013), sendo muito improvável o registro dessa família em épocas subsequentes. O fragmento de maxilar com dente foi reavaliado por Delcourt \& Grillo (2018) a partir de comparações morfológicas, análises morfométricas e filogenéticas, concluindo que o espécime UFRJ-DG409-R trata-se de um abelissaurídeo indeterminado, ao invés de carcarodontossaurídeo. Dessa forma, os autores concordaram com Canale et al. (2009) e Gianechini et al. (2015) na afirmação de que todas as coroas dentárias isoladas que já foram atribuídas aos carcarodontossaurídeos (e.g. Candeiro et al., 2006, 2012) deveriam ser criteriosamente revisadas e propuseram uma reavaliação de todas as coroas descritas para o grupo no Brasil.

O estudo amplo com diferentes métodos morfométricos e filogenéticos, como os utilizados em Delcourt \& Grillo (2018), Hendrickx \& Mateus (2014) e Hendrickx et al. (2015), vem sendo realizado pelos presentes autores e outros colaboradores (Delcourt et al., submetido) a fim de avaliar as coroas dentárias isoladas do final do Cretáceo do Brasil.
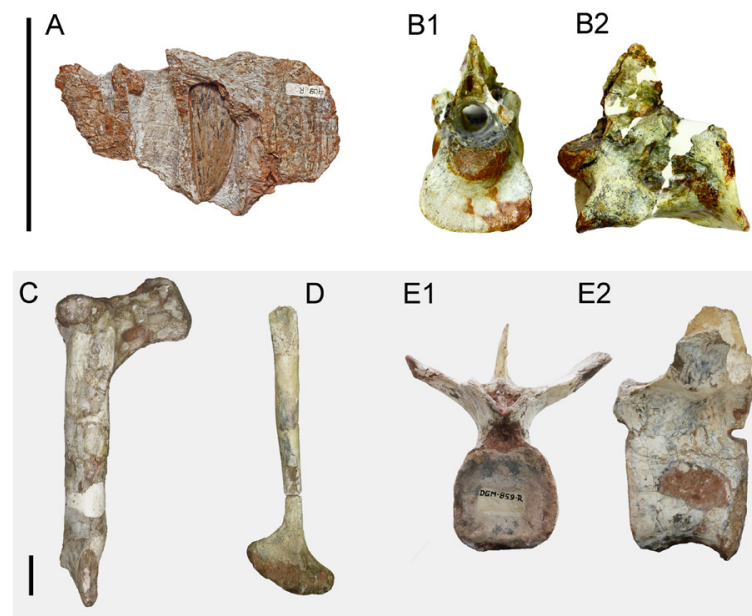

E1

E2

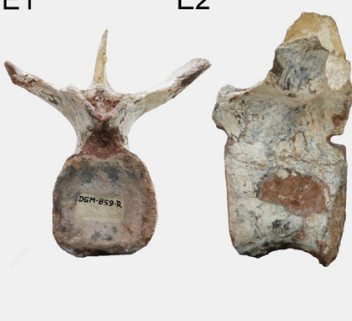

Figura 5. Elementos osteológicos de Abelissauridae recuperados em unidades geológicas brasileiras. A, UFRJ-DG409-R, fragmento de maxilar direito de abelissaurídeo indeterminado em vista lateral; B1B2, holótipo de Thanos simonattoi em vista anterior e lateral esquerda; C-E2, elementos pós-cranianos de Pycnonemosaurus nevesi; C, tíbia direita em vista lateral; D, púbis direito em vista lateral; E1-E2, vértebra caudal anterior em vista anterior e lateral esquerda. Figuras modificadas de A, Delcourt \& Grillo (2018); B1-B2, Delcourt \& Iori (2018); C-E2, Delcourt (2017). Barras de escala iguais a $10 \mathrm{~cm}$

\begin{tabular}{c|c|c|c|c|c}
\hline (C) Terrae Didat. & Campinas, SP & v.16 & $1-13$ & $\mathrm{e} 020017$ & 2020 \\
\hline
\end{tabular}


Tabela 1. Registro de Abelisauridae em diferentes unidades geológicas brasileiras. Fontes de dados: 1. Azevedo et al. (2013); 2. Bertini (19960; 3. Bittencourt \& Kellner (2002); 4. Brum et al. (2016); 5. Candeiro et al. (2004); 6. Candeiro et al. (2006a); 7. Candeiro et al. (2006b); 8. Candeiro et al. (2012); 9. Delcourt \& Grillo (2018); 10. Delcourt \& lori (2018); 11. Delcourt (2017); 12. Ghilardi \& Fernandes (2011); 13. Kellner \& Campos (2002); 14. Méndez et al. 2014); 15. Novas et al. (2008); 16. Sales et al. (2018); 17. Tavares et al. (2014)

\begin{tabular}{|c|c|c|c|c|}
\hline Táxon / Espécime & Preservação & Contexto Geológico & Datação & $\begin{array}{l}\text { Referên- } \\
\text { cia }\end{array}$ \\
\hline $\begin{array}{l}\text { Thanos simonattoi / } \\
\text { MPMA 08-0016/95 }\end{array}$ & $\begin{array}{l}\text { Axis quase completo fusio- } \\
\text { nado com um intercentro } \\
\text { axial }\end{array}$ & $\begin{array}{l}\text { Fm. São José do Rio } \\
\text { Preto (Gr. Bauru) }\end{array}$ & $\begin{array}{l}\text { Santoniano, Cre- } \\
\text { táceo Superior }\end{array}$ & 11,14 \\
\hline $\begin{array}{l}\text { Pycnonemosaurus nevesi / } \\
\text { DGM 859-R }\end{array}$ & $\begin{array}{l}\text { Esqueleto pós-craniano, } \\
\text { com duas vértebras caudais } \\
\text { incompletas,quatro centros } \\
\text { caudais, três processos } \\
\text { transversos caudais, partes } \\
\text { de uma costela, parte pró- } \\
\text { xima de um púbis direito, } \\
\text { tíbia direita, parte distal } \\
\text { da fíbula direita, além de } \\
\text { outros elementos não iden- } \\
\text { tificados. }\end{array}$ & Gr. Parecis & $\begin{array}{l}\text { Campaniano- } \\
\text {-Maastrichtiano; } \\
\text { Cretáceo Supe- } \\
\text { rior }\end{array}$ & 9,13 \\
\hline $\begin{array}{l}\text { Abelisauridae / UFRJ-D- } \\
\text { G409-R }\end{array}$ & $\begin{array}{l}\text { Uma maxila direita frag- } \\
\text { mentada com cinco al- } \\
\text { véolos e com um dente de } \\
\text { substituição }\end{array}$ & $\begin{array}{l}\text { Fm. Presidente Pruden- } \\
\text { te; Bacia Bauru }\end{array}$ & $\begin{array}{l}\text { Campaniano- } \\
\text {-Maastrichtiano; } \\
\text { Cretáceo Supe- } \\
\text { rior }\end{array}$ & 1,9 \\
\hline $\begin{array}{l}\text { Abelisauridae / UFRGS-P- } \\
\text { V-038-K }\end{array}$ & $\begin{array}{l}\text { Grande parte de uma coroa } \\
\text { dentária }\end{array}$ & $\begin{array}{l}\text { Bacia do Paraná (Unida- } \\
\text { de Cambambe) }\end{array}$ & $\begin{array}{l}\text { Cretáceo Su- } \\
\text { perior (não } \\
\text { ultrapassando o } \\
\text { Santoniano) } \\
\end{array}$ & 16 \\
\hline Abelisauridae / MN4547-V & Coroa dentária & $\begin{array}{l}\text { Formação, Alcântara Ba- } \\
\text { cia de São Luís-Grajaú }\end{array}$ & $\begin{array}{l}\text { Cenomaniano, } \\
\text { Cretáceo Supe- } \\
\text { rior }\end{array}$ & 16 \\
\hline $\begin{array}{l}\text { Abelisauridae / DGM } \\
\text { 927-R }\end{array}$ & $\begin{array}{l}\text { Parte de um ílio esquerdo } \\
\text { isolado, com as regiões } \\
\text { pré-acetabular e pós-aceta- } \\
\text { bular preservadas. }\end{array}$ & $\begin{array}{l}\text { Fm. Adamantina, Gr. } \\
\text { Bauru (sensu Soares et } \\
\text { al., 1980) }\end{array}$ & $\begin{array}{l}\text { Turoniano- } \\
\text { Maastrichtiano, } \\
\text { Cretáceo Supe- } \\
\text { rior }\end{array}$ & 4 \\
\hline MCT 1857-R & $\begin{array}{l}\text { Porção distal de um fêmur } \\
\text { direito }\end{array}$ & $\begin{array}{l}\text { Fm. Adamantina, Gr. } \\
\text { Bauru (sensu Soares et } \\
\text { al., 1980) }\end{array}$ & $\begin{array}{l}\text { Turoniano- } \\
\text { Maastrichtiano, } \\
\text { Cretáceo Supe- } \\
\text { rior }\end{array}$ & 4 \\
\hline Abelisauridae / CPP 893 & $\begin{array}{l}\text { Uma vértebra dorsal bem } \\
\text { preservada }\end{array}$ & $\begin{array}{l}\text { Fm. Marília, Bacia } \\
\text { Bauru }\end{array}$ & $\begin{array}{l}\text { Maastrichtiano, } \\
\text { Cretáceo Supe- } \\
\text { rior }\end{array}$ & 15 \\
\hline Abelisauridae / CPP 174 & $\begin{array}{l}\text { Porção distal de um fêmur } \\
\text { esquerdo }\end{array}$ & $\begin{array}{l}\text { Fm. Marília, Bacia } \\
\text { Bauru }\end{array}$ & $\begin{array}{l}\text { Maastrichtiano, } \\
\text { Cretáceo Supe- } \\
\text { rior }\end{array}$ & 15 \\
\hline Abelisauridae / CPP 692 & $\begin{array}{l}\text { Possivelmente, trata-se da } \\
\text { segunda falange do dígito } \\
\text { III }\end{array}$ & $\begin{array}{l}\text { Fm. Marília, Bacia } \\
\text { Bauru }\end{array}$ & $\begin{array}{l}\text { Maastrichtiano, } \\
\text { Cretáceo Supe- } \\
\text { rior }\end{array}$ & 15 \\
\hline Abelisauridae / URC R-44 & $\begin{array}{l}\text { Pré-maxilar direito, com } \\
\text { sessão da narina externa e } \\
\text { presença de quatro alvéolos } \\
\text { danificados, com os restos } \\
\text { de três raízes dentárias }\end{array}$ & $\begin{array}{l}\text { Fm. Adamantina, Gr. } \\
\text { Bauru (sensu Soares et } \\
\text { al., 1980) }\end{array}$ & $\begin{array}{l}\text { Turoniano- } \\
\text { Maastrichtiano, } \\
\text { Cretáceo Supe- } \\
\text { rior }\end{array}$ & $2,6,7$ \\
\hline Abelisauridae / URC R-45 & Uma coroa dentária & $\begin{array}{l}\text { Fm. Adamantina, Gr. } \\
\text { Bauru (sensu Soares et } \\
\text { al., 1980) }\end{array}$ & $\begin{array}{l}\text { Turoniano- } \\
\text { Maastrichtiano, } \\
\text { Cretáceo Supe- } \\
\text { rior }\end{array}$ & 2 \\
\hline \multicolumn{2}{|l|}{ (C) Terrae Didat. } & $1-13$ & \begin{tabular}{|l|l|} 
& $\mathrm{e} 020017$ \\
\end{tabular} & 2020 \\
\hline
\end{tabular}




\begin{tabular}{|c|c|c|c|c|}
\hline $\begin{array}{l}\text { Abelisauridae / CPP }(002, \\
020,021,121,123,124, \\
\text { 129a, 129b, 129c, 131, 132, } \\
\text { 134, 135, 136, 144, 150, } \\
\text { 154, 158, 161/1, 198, 205, } \\
206,207,208,211,242, \\
372,375 / 1,375 / 2,452,463, \\
477,446,451 / 1,452 / 1,476, \\
447,478)\end{array}$ & 38 dentes & Fm. Marília, Gr. Bauru & $\begin{array}{l}\text { Neomaastrich- } \\
\text { tiano, Cretáceo } \\
\text { Superior }\end{array}$ & $5,6,7$ \\
\hline $\begin{array}{l}\text { Abelisauridae / UFRJ-DG } \\
\text { 371-Rd; UFRJ-DG 374- } \\
\text { Rd; UFRJ-DG 378-Rd }\end{array}$ & $\begin{array}{l}\text { Três dentes (ausência da } \\
\text { extremidade apical no es- } \\
\text { pécime UFRJ-DG 371) }\end{array}$ & $\begin{array}{l}\text { Fm. Adamantina, Bacia } \\
\text { Bauru }\end{array}$ & $\begin{array}{l}\text { Santoniano- } \\
\text {-Maastrichtiano, } \\
\text { Cretáceo Supe- } \\
\text { rior }\end{array}$ & 5 \\
\hline $\begin{array}{l}\text { Abelisauridae / } \\
\text { MPMA-12-00D1-97; } \\
\text { MPMA-12-00D3-97; } \\
\text { MPMA-12-00D4-97; } \\
\text { MPMA-12-00D5-97; } \\
\text { MPMA-12-00D10-97 } \\
\text { MPMA-12-00D7-97 }\end{array}$ & $\begin{array}{l}\text { 05 dentes (MPMA-12- } \\
\text { 00D1-97, parte basal da } \\
\text { coroa dentária ausente; } \\
\text { MPMA-12-00D5-97, ápice } \\
\text { da coroa dentária ausente; } \\
\text { MPMA-12-00D7-97, base } \\
\text { da coroa dentária ausente; } \\
\text { MPMA-12-00D10-97, } \\
\text { uma pequena parte do } \\
\text { ápice da coroa dentária } \\
\text { ausente) }\end{array}$ & $\begin{array}{l}\text { Fm. Adamantina, Bacia } \\
\text { Bauru }\end{array}$ & $\begin{array}{l}\text { Santoniano- } \\
\text {-Maastrichtiano } \\
\text { (?), Cretáceo } \\
\text { Superior }\end{array}$ & 17 \\
\hline $\begin{array}{l}\text { Abelisauridae / DGM 859- } \\
\mathrm{R} \text { (é o mesmo tombo do } \\
\text { Pycnonemosaurus) }\end{array}$ & $\begin{array}{l}\text { Lote com } 09 \text { dentes iso- } \\
\text { lados }\end{array}$ & Gr. Bauru & $\begin{array}{l}\text { Campaniano- } \\
\text {-Maastrichtiano; } \\
\text { Cretáceo Supe- } \\
\text { rior }\end{array}$ & 3 \\
\hline $\begin{array}{l}\text { Abelisauridae / ??? Mor- } \\
\text { fotipo } 1\end{array}$ & 03 coroas dentárias & $\begin{array}{l}\text { Fm. Adamantina, Bacia } \\
\text { Bauru }\end{array}$ & $\begin{array}{l}\text { Santoniano- } \\
\text {-Maastrichtiano } \\
\text { (?); Cretáceo } \\
\text { Superior }\end{array}$ & 12 \\
\hline $\begin{array}{l}\text { Abelisauridae / MMR/ } \\
\text { UFU-PV } 0006\end{array}$ & 01 dente & $\begin{array}{l}\text { Fm. Adamantina, Bacia } \\
\text { Bauru }\end{array}$ & $\begin{array}{l}\text { Santoniano- } \\
\text {-Maastrichtiano, } \\
\text { Cretáceo Supe- } \\
\text { rior }\end{array}$ & 7 \\
\hline
\end{tabular}

\section{Considerações finais}

Os abelissaurídeos foram um dos grupos mais diversos e de grande sucesso evolutivo dentre os dinossauros terópodes, ocupando o topo da cadeia alimentar no final do período Cretáceo no Hemisfério Sul. Sua morfologia e distribuição por diversos continentes evidenciam seu grande sucesso evolutivo desde o Jurássico. Ainda assim, é necessária una intensa pesquisa para compreender questões evolutivas que estão em aberto. Algumas dessas questões são: (i) os abelissaurídeos estavam distribuídos em outros continentes do Hemisfério Norte além de Europa?; (ii) por que os abelissaurídeos tinham tendência em desenvolver apêndices cranianos?; (iii) o desenvolvimento dessas estruturas está relacionado à extrema redução dos membros anteriores? e (iv) qual foi o mecanismo evolutivo que levou a redução dos braços, este seria comum a outros terópodes, como Tyrannosaurus? Essas e outras questões podem e devem nortear o estudo dos abelissaurídeos, bem como incentivar aos pesquisadores a coleta em afloramentos com probabilidade de resgate de seus fósseis.

No caso do Brasil, nós concordamos com Bittencourt \& Langer (2011) de que a escassez de abelissaurídeos e outros fósseis entre o Aptiano e o Cenomaniano, se dá pela provável falta de afloramentos em função da cobertura vegetal. Cabe ressaltar também que, no Grupo Bauru, o registro de dinossauros de maneira geral é comprometido pelo paleoambiente de deposição, conforme demonstrado por Bandeira et al. (2018). Contudo, o fato da maioria do registro ser de origem dentária, não impede que esses fósseis forneçam informações acerca da ecologia e taxonomia de Abelisauridae (Delcourt \& Grillo, 2018). 


\section{Agradecimentos}

Os autores agradecem a Pedro Rodrigues Busana pela disponibilização da Figura 4. Os autores agradecem a Coordenação de Aperfeiçoamento de Pessoal de Nível Superior (CAPES) pelo suporte financeiro de PNPD, bolsa de doutorado e ao Concelho Nacional de Pesquisa (CNPq) e pela bolsa de produtividade em pesquisa (PQ).

\section{Referências}

Agnolin, F. L., \& Chiarelli, P. (2010). The position of the claws in Noasauridae (Dinosauria: Abelisauroidea) and its implications for abelisauroid manus evolution. Palaontologische Zeitschrift, 84: 293-300. doi: 10.1007/s12542-009-0044-2.

Azevedo, R. P. F., Simbras, F. M., Furtado, M. R., Candeiro, C. R. A., \& Bergqvist, L. P. (2013). First Brazilian carcharodontosaurid and other new theropod dinosaur fossils from the Campanian-Maastrichtian Presidente Prudente Formation, São Paulo State, southeastern Brazil. Cretaceous Research, 40, 131-142. doi: 10.1016/j.cretres.2012.06.004.

Bandeira, K. L., Brum, A. S., Pêgas, R. V., Cidade, G. M., Holgado, B., Cidade, A., \& Souza, R. G. (2018). The Baurusuchidae vs Theropoda record in the Bauru Group (Upper Cretaceous, Brazil): a taphonomic perspective. Journal of Iberian Geology, 44(1), 25-54. doi: 10.1007/s41513-018-0048-4.

Bertini, R. J. (1996). Evidências de Abelisauridae (Carnosauria: Saurischia) do Neocretáceo da Bacia do Paraná. In: Simpósio sobre o Cretáceo do Brasil, 4, pp. 267-271.

Bittencourt, J. D. S., \& Kellner, A. W. A. (2002). Abelisauria (Theropoda, Dinosauria) teeth from Brazil. Boletim do Museum Nacional, 68, 1-8.

Bittencourt, J. S., \& Langer, M. C. (2011). Mesozoic dinosaurs from Brazil and their biogeographic implications. Anais da Academia Brasileira de Ciências, 83, 23-60. doi: 10.1590/S0001-37652011000100003.

Bonaparte, J. F., \& Novas, F. (1985). Abelisaurus comahuensis, n.g., n.sp., Carnosauria del Crétacico Tardio de Patagonia. Ameghiniana, 21(2-4), 259-265.

Bonaparte, J. F. (1991). The Gondwanian theropod families Abelisauridae and Noasauridae. Historical Biology, 5(1), 1-25. doi: 10.1080/10292389109380385.

Bonaparte, J. F. (1985). A horned Cretaceous carnosaur from Patagonia. National Geographic Research, 1,149-151.

Bonaparte, J. F., Novas, F. E., \& Coria, R. A. (1990). Carnotaurus sastrei Bonaparte, the horned, lightly built carnosaur from the Middle Cretaceous of Patagonia. Natural History Museum of Los Angeles County, 1-42.
Brochu, C. A. (2003). Osteology of Tyrannosaurus rex: insights from a nearly complete skeleton and high-resolution computed tomographic analysis of the skull. Journal of Vertebrate Paleontology, 22, 1-138. doi: 10.1080/02724634.2003.10010947.

Brum, A. S., Machado, E. B., Campos, D. A., \& Kellner, A. W. A. (2016). Morphology and internal structure of two new abelisaurid remains (Theropoda, Dinosauria) from the Adamantina Formation (Turonian - Maastrichtian), Bauru Group, Parana Basin, Brazil. Cretaceous Research, 60, 287-296. doi: 10.1016/j.cretres.2015.11.013.

Burch, S. H. (2017). Myology of the forelimb of Majungasaurus crenatissimus (Theropoda, Abelisauridae) and the morphological consequences of extreme limb reduction. Journal of Anatomy, 231, 515-531. doi: 10.1111/joa.12660.

Burch, S. H., \& Carrano, M. T. (2012). An articulated pectoral girdle and forelimb of the abelisaurid theropod Majungasaurus crenatissimus from the Late Cretaceous of Madagascar. Journal of Vertebrate Paleontology, 32, 1-16. doi: 10.1080/02724634.2012.622027.

Canale, J. I., Apesteguía, S., Gallina, P. A., Gianechini, F. A., \& Haluza, A. (2017). The oldest theropods from the Neuquén Basin: Predatory dinosaur diversity from the Bajada Colorada Formation (Lower Cretaceous: Berriasian-Valanginian), Neuquén, Argentina. Cretaceous Research, 71, 6378. doi: 10.1016/J.CRETRES.2016.11.010.

Canale, J. I., Scanferla, C. A., Agnolin, F. L., \& Novas, F. E. (2009). New carnivorous dinosaur from the Late Cretaceous of NW Patagonia and the evolution of abelisaurid theropods. Naturwissenschaften, 96, 409-414. doi: 10.1007/s00114-008-0487-4.

Candeiro, C. R. A., Abranches, C. T., Abrantes, E. A., Avilla, L. S., Martins, V. C., Moreira, A. L., Torres, S. R., \& Bergqvist, L. P. (2004). Dinosaur remains from Western São Paulo State, Brazil (Bauru Basin, Adamantina Formation, Upper Cretaceous). Journal of South American Earth Science, 18, 1-10. doi: 10.1016/j.jsames.2004.08.004.

Candeiro, C. R. A., Martinelli, A. G., Avilla, L. S., \& Rich, T. H. (2006). Tetrapods from the upper Cretaceous (Turonian-Maastrichtian) Bauru group of Brazil: a reappraisal. Cretaceous Research, 27(6), 923946. doi: 10.1016/j.cretres.2006.05.002.

Candeiro, C. R. A., Santos, A. R., Rich, T. H., Marinho, T. S., \& Oliveira, E. C. (2006b). Vertebrate fossils from the Adamantina Formation (Late Cretaceous), Prata Paleontological District, Minas Gerais State, Brazil. Geobios, 39, 319-327. doi: 10.1016/j.geobios.2005.10.003.

Candeiro, C. R. A., Currie, P. J., \& Bergqvist, L. P. (2012). Theropod teeth from the Marília Formation (late Maastrichtian) at the paleontological site of Peirópolis in Minas Gerais State, Brazil. Re- 
vista Brasileira de Geociências, 42(2), 323-330. doi: 10.5327/Z0375-75362012000200008.

Carpenter, C. C. (1967). Aggression and social structure in iguanid lizards. In: Lizard Ecology: A Symposium. pp. 87-105.

Carr, T. D., Varricchio, D. J., Sedlmayr, J. C., Roberts, E. M., \& Moore, J. R. (2017). A new tyrannosaur with evidence for anagenesis and crocodile-like facial sensory system. Scientific Reports, 7, 44942. doi: 10.1038/srep44942.

Carrano, M. T. (2007). The Appendicular Skeleton of Majungasaurus Crenatissimus (Theropoda: Abelisauridae) From the Late Cretaceous of Madagascar. Journal of Vertebrate Paleontology, 27, 163-179. doi: 0.1671/0272-4634(2007)27[163:TASOMC]2.0. $\mathrm{CO} ; 2$.

Carrano, M. T., Loewen, M. A., \& Sertich, J.J. W. (2011). New materials of Masiakasaurus knopfleri Sampson, Carrano, and Forster, 2001, and implications for the morphology of the Noasauridae (Theropoda: Ceratosauria). Smithsonian contributions to paleobiology, 95, 1-53. doi: 10.5479/si.00810266.95.1.

Carrano, M. T., \& Sampson, S. D. (2008). The Phylogeny of Ceratosauria (Dinosauria: Theropoda). Journal of Systematic Palaeontology, 6, 183-236. doi: 10.1017/S1477201907002246.

Coria, R. A., \& Salgado, L. (1995). A new giant carnivorous dinosaur from the Cretaceous of Patagonia. Nature, 377, 224-226. doi: 10.1038/377224a0.

Coria, R. A., Chiappe, L. M., \& Dingus, L. (2002). A new close relative of Carnotaurus sastrei Bonaparte 1985 (Theropoda: Abelisauridae) from the Late Cretaceous of Patagonia. Journal of Vertebrate Paleontology, 22, 460-465. doi: $\quad 10.1671 / 0272-4634(2002) 022[0460: A N-$ CROC]2.0.CO;2.

Coria, R. A., \& Salgado, L. (2005). Mid-Cretaceous turnover of saurischian dinosaur communities: evidence from the Neuquén Basin. The Geological Society of London, 252(1), 317-327. doi: 10.1144/gsl. sp.2005.252.01.16.

Delcourt, R. (2018). Ceratosaur palaeobiology: New insights on evolution and ecology of the southern rulers. Scientific Reports, 8, 9730. doi: 10.1038/ s41598-018-28154-x.

Delcourt, R. (2017). Revised morphology of Pycnonemosaurus nevesi Kellner \& Campos, 2002 (Theropoda: Abelisauridae) and its phylogenetic relationships. Zootaxa, 4276, 1-45. doi: 10.11646/ zootaxa.4276.1.1.

Delcourt, R. (2016). Evolução morfológica de Ceratosauria e Tyrannosauroidea (Dinosauria: Theropoda). São Paulo: Inst. Bio. Universidade de São Paulo. 227p. (Tese Dout.).

Delcourt, R., \& Grillo, O. N. (2018). Reassessment of a fragmentary maxilla attributed to Carcharodontosauridae from Presidente Prudente Forma- tion, Brazil. Cretaceous Research, 84, 515-524. doi: 10.1016/j.cretres.2017.09.008.

Delcourt, R., \& Iori, F. V. (2018). A new Abelisauridae (Dinosauria: Theropoda) from São José do Rio Preto Formation, Upper Cretaceous of Brazil and comments on the Bauru Group fauna. Historical Biology, 1-8. doi: 10.1080/08912963.2018.1546700.

Filippi, L. S., Méndez, A. H., Valieri, R. D. J., \& Garrido, A. C. (2016). A new brachyrostran with hypertrophied axial structures reveals an unexpected radiation of latest Cretaceous abelisaurids. Cretaceous Research, 61, 209-219. doi: 10.1016/j.cretres.2015.12.018.

Ghilardi, A. M., \& Fernandes, M. A. (2011). Dentes de Theropoda da Formação Adamantina (Cretáceo Superior, Bacia Bauru) da região do município de Ibirá, São Paulo, Brasil. In: Calvo J. O., Porfiri J., Riga B.G., \& dos Santos D. ed. (2011). Paleontología y Dinosaurios desde América Latina. EDIUNC. (pp. 115-123).

Gianechini, F. A., Apesteguia, S., Landini, W., Finotti, F., Valieri, R. D. J., \& Zandonai, F. (2015). New abelisaurid remains from the Anacleto Formation (Upper Cretaceous), Patagonia, Argentina. Cretaceous Research, 54, 1-16. doi: 10.1016/j.cretres.2014.11.009.

Grillo, O. N., \& Delcourt, R. (2017). Allometry and body length of abelisauroid theropods: Pycnonemosaurus nevesi is the new king. Cretaceous Research, 69, 71-89. doi: 10.1016/j.cretres.2016.09.001.

Hendrickx, C., \& Mateus, O. (2014). Abelisauridae (Dinosauria: Theropoda) from the Late Jurassic of Portugal and dentition-based phylogeny as a contribution for the identification of isolated theropod teeth. Zootaxa, 3759 (1), 1-74. doi: 10.11646/ zootaxa.3759.1.1.

Hendrickx, C., Mateus, O., \& Araújo, R. (2015). A proposed terminology of theropod teeth (Dinosauria, Saurischia). Journal of Vertebrate Paleontology, e982797, 1-18. doi: 10.1080/02724634.2015.982797.

Hieronymus, T. L., Witmer, L. M., Tanke, D. H., \& Currie, P. J. (2009). The facial integument of centrosaurine ceratopsids: morphological and histological correlates of novel skin structures. The Anatomical Record, 292, 1370-1396. doi: 10.1002/ ar.20985.

Kellner, A. W. A., \& Campos, D. A. (2002). On a new theropod dinosaur (Abelisauria) from the continental Cretaceous of Brazil. Arquivos do Museu Nacional, 60, 163-170.

Langer, M. C., Ezcurra, M. D., Rauhut, O. W. M., Benton, M. J., Knoll, F., McPhee, B. W., Novas, F. E., Pol, D., \& Brusatte, S. L. (2017). Untangling the dinosaur family tree. Nature, 551(E1), 1-5. doi: $10.1038 /$ nature24012.

Le Loeuff, J., \& Buffetaut, E. (1991). Tarascosaurus sallu-

\begin{tabular}{c|c}
\hline (C) Terrae Didat. & Campinas, SP \\
\hline
\end{tabular} 
vicus, new genus, new species, a theropod dinosaur from the Upper Cretaceous of southern France. Geobios, 24, 585-594.

Lockley, M., Kukihara, R., \& Mitchell, L. (2008). Why Tyrannosaurus rex had puny arms: an integral morphodynamic solution to a simple puzzle in theropod paleobiology. In: Larson P.L., Carpenter K. (ed). Tyrannosaurus Rex, the Tyrant King (pp. 130164) Indiana: University Press, Bloomington.

Longrich, N. R., Pereda-Suberbiola, X., Jalil, N. E., Khaldoune, F., \& Jourani, E. (2017). An abelisaurid from the latest Cretaceous (late Maastrichtian) of Morocco, North Africa. Cretaceous Research, 76, 40-52. doi: 10.1016/j.cretres.2017.03.021.

Madsen Jr, J. H. (1976). Allosaurus fragilis: a revised osteology. Utah Geological and Mineral Survey, Bulletin, 109, 1-163.

Mazzetta, G. V., Cisilino, A. P., Blanco, R. E., \& Calvo, N. (2009). Cranial mechanics and functional interpretation of the horned carnivorous dinosaur Carnotaurus sastrei. Journal of Vertebrate Paleontology, 29, 822-830. doi: 10.1671/039.029.0313.

Méndez, A. H., Novas, F. E., \& Iori, F. V. (2012). First record of Megaraptora (Theropoda, Neovenatoridae) from Brazil. Comptes Rendus Palevol, 11(4), 251-256. doi: 10.1016/j.crpv.2011.12.007.

Méndez, A. H. (2014). The cervical vertebrae of the Late Cretaceous abelisaurid dinosaur Carnotaurus sastrei. Acta Palaeontologica Polonica, 59(3), 569-579. doi: https://doi.org/10.4202/app.2011.0129.

Méndez, A. H., Novas, F. E., \& Iori, F. V. (2014). New record of abelisauroid theropods from the Bauru Group (Upper Cretaceous), São Paulo State, Brazil. Revista Brasileira de Paleontologia, 17, 23-32. doi: 10.4072/rbp.2014.1.03.

Novas, F. E., Valais, S., Vickers-Rich, P., \& Rich, T. (2005). A large Cretaceous theropod from Patagonia, Argentina, and the evolution of carcharodontosaurids. Naturwissenschaften, 92 (5), 226-230. doi: 10.1007/s00114-005-0623-3.

Novas, F. E., Carvalho, I. S., Ribeiro, L. C. B., \& Méndez, A. H. (2008). First abelisaurid bone remains from the Maastrichtian Marília Formation, Bauru Basin, Brazil. Cretaceous Research, 29, 625-635. doi: 10.1016/j.cretres.2008.01.010.

Novas, F. E., Agnolín, F. L., Ezcurra, M. D., Porfiri, J., \& Canale, J. I. (2013). Evolution of the carnivorous dinosaurs during the Cretaceous: The evidence from Patagonia. Cretaceous Research, 45, 174215. doi: 10.1016/j.cretres.2013.04.001.

O'Connor, P. M. (2007). The postcranial axial skeleton of Majungasaurus crenatissimus (Theropoda: Abelisauridae) from the Late Cretaceous of Madagascar. Journal of Vertebrate Paleontology, 27, 127-163. doi: 10.1671/0272-4634(2007)27.

Paul, G. S. (2010). The Princeton Field Guide to Dinosaurs. Princeton University Press.
Persons, W. S., \& Currie, P. J. (2011). Dinosaur speed demon: The caudal musculature of Carnotaurus sastrei and implications for the evolution of South American abelisaurids. PLoS One, 6(e25763), 1-11. doi: 10.1371/journal.pone.0025763.

Pol, D., \& Rauhut, O. W. M. (2012). A Middle Jurassic abelisaurid from Patagonia and the early diversification of theropod dinosaurs. Proceedings of the Royal Society B, 279, 1-6. doi: 10.1098/rspb.2012.0660.

Rauhut, O. W. M., \& Carrano, M. T. (2016). The theropod dinosaur Elaphrosaurus bambergi Janensch, 1920, from the Late Jurassic of Tendaguru, Tanzania. Zoological Journal of the Linnean Society, 178, 546-610. doi: 10.1111/zoj.12425.

Rogers, R. R., Krause, D. W., Rogers, K. C., Rasoamiaramanana, A. H., \& Rahantarisoa, L. (2007). Paleoenvironment and paleoecology of Majungasaurus crenatissimus (Theropoda: Abelisauridae) from the Late Cretaceous of Madagascar. Journal of Vertebrate Paleontology, 27, 21-31. doi: 10.1671/0272-4634(2007)27[21:PAPOMC]2.0. $\mathrm{CO} ; 2$.

Romer, A. S. (1923). Crocodilian pelvic muscles and their avian and reptilian homologues. Bulletin of the American Museum of Natural History, 48, 533-552.

Sakamoto, M. (2010). Jaw biomechanics and the evolution of biting performance in theropod dinosaurs. Proceedings of the Royal Society B, 277, 3327-3333. doi: 10.1098/rspb.2010.0794.

Sales, M. A. F., Oliveira, I. A. P., \& Schultz, C. L. (2018). The oldest abelisaurid record from Brazil and the palaeobiogeographic significance of mid-Cretaceous dinosaur assemblages from northern South America. Palaeogeography, Palaeoclimatology, Palaeoecology, 508, 107-115. doi: 10.1016/j. palaeo.2018.07.024.

Sampson, S. D., Witmer, L. M., Forster, C. A., Krause, D. W., O'Connor, P. M., Dodson, P., \& Ravoavy, F. (1998). Predatory dinosaur remains from Madagascar: Implications for the Cretaceous biogeography of Gondwana. Science, 280, 1048-1051. doi: 10.1126/science.280.5366.1048.

Sampson, S. D., \& Witmer, L. M. (2007). Craniofacial Anatomy of Majungasaurus Crenatissimus (Theropoda: Abelisauridae) From the Late Cretaceous of Madagascar. Journal of Vertebrate Paleontology, 27, 32102. doi: 10.1671/0272-4634(2007)27[32:CAOMCT]2.0.CO;2.

Sereno, P. C. (1999). The Evolution of Dinosaurs. Science, 284(5423), 2137-2147. doi: 10.1126/science.284.5423.2137.

Sereno, P. C., Wilson, J. A., \& Conrad, J. L. (2004). New dinosaurs link southern landmasses in the Mid-Cretaceous. Proceedings of the Royal Society B, 271, 1325-1330. doi: 10.1098/rspb.2004.2692.

Snively, E. (2006). Neck musculoskeletal function in the Tyrannosauridae (Theropoda, Coelurosauria): implications 
for feeding dynamics. Alberta: University of Calgary, Calgary. (Ph.D. thesis).

Snively, E., Cotton, J. R., Witmer, L. M., Ridgely, R., \& Theodor, J. (2011). Finite Element Comparison of Cranial Sinus Function in the Dinosaur Majungasaurus and Head-Clubbing Giraffes, in: ASME 2011 Summer Bioengineering Conference. pp. 1071076.

Snively, E., \& Theodor, J. M. (2011). Common Functional Correlates of Head-Strike Behavior in the Pachycephalosaur Stegoceras validum (Ornithischia, Dinosauria) and Combative Artiodactyls. PLoS One, 6(e21422), 1-26. doi: 10.1371/journal. pone.0021422.

Tanke, D. H., \& Currie, P. J. (1998). Head-biting behavior in theropod dinosaurs: Paleopathological evidence. GAIA-Ecological Perspectives for Science and
Society, 184, 167-184. doi: 10.7939/R34T6FJ1P.

Tavares, S. A. S., Branco, F. R., \& Santucci, R. M. (2014). Theropod teeth from the Adamantina Formation (Bauru Group, Upper Cretaceous), Monte Alto, São Paulo, Brazil. Cretaceous Research, 50, 59-71. doi: 10.1016/j.cretres.2014.03.021.

Tortosa, T., Buffetaut, E., Vialle, N., Dutour, Y., Turini, E., \& Cheylan, G. (2014). A new abelisaurid dinosaur from the Late Cretaceous of southern France: Palaeobiogeographical implications. $A n-$ nales de Paléontologie, 100 (1), 63-86. doi: 10.1016/j. annpal.2013.10.003.

Wang, S., Stiegler, J., Amiot, R., Wang, X., Du, G. H., Clark, J. M., \& Xu, X. (2017). Extreme Ontogenetic Changes in a Ceratosaurian Theropod. Current Biology, 27, 144-148. doi: 10.1016/j. cub.2016.10.043. 